\title{
The Effect of Artificial Intelligence on the Human Idea of Free Will
}

\begin{abstract}
Eric Luo*
The Webb Schools in Claremont, California, USA

*Corresponding author. Email: eluo@webb.org

ABSTRACT

Artificial Intelligence (AI) has made advances in its ability to mimic and surpass human problem solving and decision-making abilities. As AI technology advances, the public begins to use AI products for their own personal decision-making. However, AI products may encounter the problem of humans' fondness of free will. Human free will is traditionally conceived of as a kind of power to control one's choices and actions. This research attempts to find out how humans react to AI decision-making products, discover the underlying reasons relating to human free will, and discuss future trends. Not only does this research help to give guidance to the development of AI products that would be accepted within different human groups, but it also helps to understand how decision-making AI may affect human culture and human society. In order to glean this, we have split this study into four parts. These are about what decisions AI already make for humans, what they could possibly make in the future, how different people view decision-making AI, and finally how humans may react to AI in general. This research starts from an analysis of the paradox of choice, which guides that, in certain circumstances, less choice may lead to more satisfaction during the decision-making process. In addition to the research about AI decision-making and the paradox of choice, surveys of 17 people were conducted as well as interviews of four individuals from different age and cultural groups. The analysis showed a strong correlation between the age and cultural backgrounds and the responses to the acceptance of AI decision-making. The conclusion of the investigation shows that in the future a more pronounced paradox of choice would occur. While people stating that they value free will, breaking it down may show that people don't really need free will to be happy if the result is satisfactory. There was large disagreement as to whether AI decisionmaking is positive or negative in human society. Future studies may do interviews and surveys at a larger scale to glean more significant information and more research can be done into the ethical considerations of how humans value free will.
\end{abstract}

Keywords: Artificial Intelligence, Human free Will, Paradox of Choice, AI Decision Making, Choice overload, AI's Effect on Humans' free will, AI's influence on human lives, Future trends in AI, User Profiling, AI Apps, AI Products.

\section{INTRODUCTION}

In recent times, Artificial Intelligence (AI) has made great advances in its ability to mimic human problemsolving and decision-making skills. Certain examples currently include applications in law firms, detecting financial fraud and making business decisions. AI has been increasingly helping humans make decisions and, in some cases, make decisions for humans. It has also been shown that AI is superior to humans in certain decision-making, like those relevant to the stock market.
In the future and arguably in the present, there may be additional advances in AI technology that will allow it to break into the mainstream and allow more people to utilize the decision-making abilities of AI for personal use. This AI could use data available in an increasingly connected world to make decisions based on enormous amounts of training data gleaned from the internet. At this point, larger tasks such as determining good career paths and helping with socialization may be done by AI.

If AI starts controlling people's lives, playing the game of life better than humans can, a question of free will emerges. Many of the decisions which humans make will now be made by something else, which may 
lead to people feeling losing freedom. In general, humans inherently like free will. If AI takes it away, there may be problems relating to humans feeling anxiety and depression.

To see this issue in action, chess can be analyzed. In 1997, IBM's Deep Blue computer defeated reigning champion Gary Kasparov, in a highly publicized match. After that, more powerful chess engines were developed, including Stockfish, which is currently one of the most powerful chess engines. However, even though humans have been rendered totally obsolete in chess, humans still enjoy chess and play it. In fact, the engines have become used more as a tool for making humans enjoy chess more. Chess engines have been banned as their use is considered cheating in many chess circles [1]. Chess was and is still relegated to human affairs where humans play as a form of leisure. People did not see a large amount of anxiety and depression from the AI being better than them and replacing their decision-making. However, this is because chess is a game where people play others to challenge themselves and have fun rather than solely winning. As $\mathrm{Mr}$. Wagonfuhr said, "If the AI knows exactly how your traps work and how to prevent them, it makes the game uninteresting in comparison to the drama and excitement of watching the opponents' eyes look at the pieces, hoping he didn't see your trap."

People's lives are different, and "winning" is the end result for most people. However, it should be kept in mind that humans could start seeing life as a game, not for survival and luxury but for fun. This is because an AI dominated world could optimize the supply chain allowing people to live longer and more luxuriously in spite of any decisions that they make.

The question is whether humans will surrender control of their own lives to AI or reject AI completely. If humans refuse to submit to AI, AI may need to include choice in its design, illusory or real, to make appropriate decisions. That question can be further subdivided into four additional questions.

1. What human decisions are already being made or influenced by AI?

2. What AI could make human decisions in the future?

3. What is the opinion of people from different age groups or cultural backgrounds on using AI to make decisions concerning smaller and larger issues such as career paths and relationships?

4. Based on the current psychological and sociological trends, how may humans react to AI taking away free will?

\section{THE PARADOX OF CHOICE}

In terms of determining how humans may react to AI making decisions for them, it is important to see how humans feel about choice and freedom.

The term "Paradox of Choice" was coined by Barry Schwartz in his 2004 book, which covered this topic [2]. This book combines studies from different areas of psychology. Schwartz describes the way humans often feel more stress and anxiety when given more choice. When describing "why we suffer", he says that choices that were once made by family, the community, and the workplace, are now being done on an individual basis which puts extra pressure on people. He also writes that missed opportunities cause stress since every decision will have trade-offs. If one makes decision A, they may be unhappy with the trade-offs involved even though decision B may have other trade-offs which they haven't considered.

In 2000, a landmark paper released by Sheena Iyengar and Mark Lepper showed the paradox of choice in an experimental form [3]. Two shops were set up in the "Jam Experiment", both selling jams of different flavors. One shop had only 6 choices while the other shop had 30 choices. Out of the two shops, the shop with more choices had $50 \%$ more people enter it than the shop with fewer choices. However, only $3 \%$ of those who entered the shop with more choices made a purchase compared to $30 \%$ in the shop, which had fewer choices. Another significant statistic deduced from this study is the average number of jams flavors sampled by the customers of both shops: 1.4 for the shop with less choice and 1.5 for the shop with more. This shows that oftentimes, the human psyche prefers less choice even though they think they will be happier with more choice. Hence the shop with more choice had more customers. The hypothesis discussed in the study was that the immensely large number of choices paralyzes people to pick from and decide not to choose at all. This is supported by the fact that the number of samples taken by each customer was similar, suggesting that those in the shop with more choices didn't have enough information to decide which one they liked the most.

There have been many attempts to duplicate the paradox of choice in numerous studies. This has had mixed success with large variances that suggest that choice overload and the paradox of choice could be tied to certain pre-conditions.

A 2015 meta-analysis by Alexander Chernev attempted to identify those pre-conditions [4]. It was shown that the complexity of the decision, the difference in choice, the amount of uncertainty or knowledge the decision maker has when processing the benefits and drawbacks of each choice, and the extent to which the decision maker aims to minimize the cognitive effort required are reliable proportional 
predictors of choice overload and the paradox of choice. The situations in which people minimize cognitive effort was described in the meta-analysis as including decision intent (buying or browsing), decision focus (choosing a group or an individual option), and the level of construal. In other words, the more complicated it is to make the choices, the more likely it is that people will feel overwhelmed. In this meta-analysis, choice overload was measured by the amount of satisfaction and regret reported, the amount of choice deferral, and the amount of switching.

In conclusion, the paradox of choice shows that humans are often paralyzed and stressed by large amounts of choice, especially when it is complicated to consider every choice's drawback and benefit. This creates a more pressurized decision-making process.

\section{HYPOTHESIS}

Based on the knowledge about the paradox of choice, it is hypothesized in this paper that humans will not be opposed to AI making decisions for them and that AI may lead to a higher standard of living and stress reduction.

In terms of how different age and cultural backgrounds may affect people's opinions, it is hypothesized that younger individuals will be more opposed to the idea of their free will being taken because, in general, younger people strive for freedom from society and people of authority such as teachers or parents. In terms of cultural opinions, it is hypothesized that there will be a negligible effect. However, there may be a slight correlation between a western background and an opposition to AI taking free will.

\section{METHODOLOGY}

To determine what decisions AI has already started making for humans, library research and fieldwork were conducted in the form of interviews and observations relating to the everyday decisions of an individual. Notes were written every time a decision was made in reference to or prompted by an AI or computer algorithm

To assess what decisions AI may be able to make in the future, library research examined the opinions of current futurologists and the technologies that already exist, some well-established and others in early development. In addition, interviewees were asked to share their opinions on future possibilities.

Four interviews were conducted to ascertain how age and culture affect people's opinions on the use of AI in career planning, relationships, and smaller daily decisions. The interviewees were a teenager with a western background, a teenager with a Chinese background, an adult with a western background, and an adult with a Chinese background. These interviews were done in a semi-structured format. The interview began by asking the participant to what extent they valued their free will and why. After that, they were introduced to the topic of free will and encouraged to converse freely with the interviewer in relation to this. To follow, the interviewer presented the participant with different scenarios and asked them their opinions. Each scenario involved a decision, and they progressively got more significant. The scenarios included the use of AI in picking restaurants, the use of AI for playing chess competitively, the use of AI in choosing a career, and the use of AI in social and relationship decisions. The variety of scenarios given reflects the different ways in which AI decision-making may affect humans. The interviewees are encouraged to ask questions throughout.

On top of these interviews, 17 individuals took part in a survey. The questions were closed versions of the interview questions. The surveys were used to find trends and correlations, while the interviews were used to justify them.

Aside from that, a case study was done on the paradox of choice, which was done using jelly beans bought at a local supermarket. These jelly beans were used as an incentive for the surveys and interviews. There were five different flavors of jelly beans in the packet. A third of the test subjects were allowed to choose between all five flavors, another third were only given two choices, and another third were given no choice on the flavor whatsoever. The test subjects weren't told what each jelly bean's flavor was and were allowed to ask what the flavors were. The test subjects were then surveyed on how satisfied they were with their choice. They were also asked whether they would or wouldn't want to choose the same flavor again.

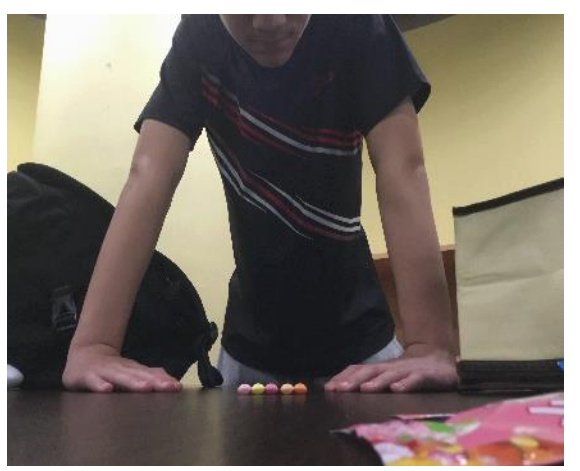

Figure 1. Jelly Bean Experiment

To consider how humans in general will react to AI in the future, a general analysis was done on all of the information gleaned from the previous methods of research. 


\section{ANALYSIS}

\subsection{What decisions have AI already started making for humans?}

Currently, AI usually uses machine learning to perform tasks. They use training data to adjust their parameters to function appropriately for their assigned task. This kind of AI already does many things better than humans. Companies have used AI to collect more personal data than what is humanly possible, sometimes more than an individual's family and friends know about them. For example, in 2012, popular retailer Target sent advertisements and coupons for baby products in the mail to a teenage girl [5]. Her father ran into the local Target in a rage, asking whether Target was trying to get his daughter pregnant. However, Target wasn't trying to encourage her to become pregnant. She was already pregnant. Target had used AI algorithms to tailor their marketing campaigns to relevant consumers. These algorithms used data gained from the teenage girl's shopping choices to determine that she was pregnant. They found that she was buying certain products such as unscented lotion and soaps and cotton balls, which were correlated with pregnancy. Target's algorithms connected this with pregnancy and suggested the baby products as ideal items to sell to the girl in question.

There are already a huge number of decisions made by AI right now. This study mostly studies the implications of AI in individual lives rather than for business, so the examples examined in this study will be predominantly based on individuals.

One of the most publicized ways that AI has affected humans is in recommending media for people. As a famous example, the YouTube "algorithm" decides what videos are recommended on the front page of YouTube for different people. It looks at what people have clicked on before and what they are most engaged by to determine what kinds of videos they highlight to a user. This has been proven to be extremely effective. Another example is the Chinese app, Toutiao, which recommends personalized articles to people based on their interests. These applications are often very addictive and can lead to a reliance on AI to stimulate pleasure in the brain. One adult subject said during the interview, "Toutiao makes it hard to steer away from what it recommends for you. The more you use it, the narrower and homogenous it becomes. When I first started using it, I hated how it didn't give me a chance to choose what I wanted to watch, but eventually, I surrendered." Because of human psychology and passiveness, people will often click on whatever is recommended instead of searching the wide variety of choices available in a place such as a library. Even if an individual wants to, they may give in to passiveness because of the immense psychological triggers employed in these apps.

Another example is the use of AI to help choose a restaurant or food venue. Apps such as Yelp or Dianping in China take into account the user's previous preferences, the frequency of visits or orders at a restaurant, the location of the user, and the reviews the user gives to determine the restaurants they recommend. These kinds of apps often use advertisements paid for by the restaurants that want to encourage new customers. While the human still has a choice, certain psychological cues such as the order, the number of stars, or other distinguishing marks can all drastically increase or decrease the chances of an individual choosing a certain restaurant.

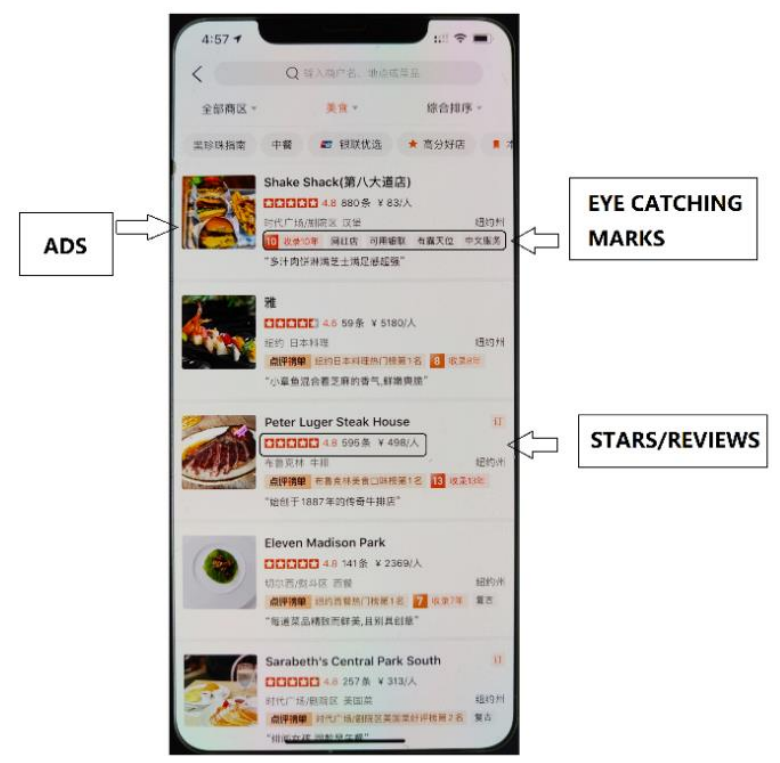

Figure 2. Examples of Psychological Triggers on the Popular Chinese Food Review Service, Dianping

AI has also helped people determine which college or college major to study. There is a myriad of online tools to help with that choice. As an example, The Council of University Presidents of Thailand has developed an AI system to analyze user interests and match them with university majors [6]. What is interesting about the goals of this $\mathrm{AI}$ is that the developer, Mr. Suchatvee, said, "For many Thai kids, parents play a major role in their decision making about careers and study. Students need guidance while they navigate a difficult series of choices. The kindest thing is to support them rather than taking those choices away." He believes that giving children support when making such important decisions is better than having parents decide.

Numerous decisions AI currently makes instead of humans. These other decisions include the selection of dating partners, the picking of houses to rent or buy, and the picking of movies. In the business world, there are also decisions such as firing and hiring for jobs. Many 
start-ups are working on AI, which can replace middlemanagement positions. It usually uses AI to determine which tasks can be done by an AI and which tasks require internet freelancers. The $\mathrm{AI}$ decides who to hire and who to fire. While the workers work, the AI learns from them, slowly learning how to do the task. In fact, one study predicted that AI will do $69 \%$ of managers' work by 2024 [7]. On the other side of the college admissions system, many college admission offices are employing AI to filter out unqualified applicants. In general, many decisions are already being advised on or influenced by AI, though there aren't many decisions done entirely by AI yet.

\subsection{What decisions could AI make in the future?}

In the future, AI will make further advancements that will enable them to make more decisions on behalf of humans, perhaps even replacing the need for human support altogether. AI doesn't need to be perfect. They just have to be close to or better than humans.

For AI to make further progress, certain milestones need to be achieved. The examples to be discussed here involve the AI's ability to have the theory of mind and organise data without humans' supervision.

The theory of mind is defined as imagining other people's thoughts, understanding that they know and feel different things. Human children usually develop this at around the age of four. A test called the SallyAnne test determines whether children have developed this skill [8]. Basically, there is one box and one basket. Sally puts a cookie in the box. Sally leaves, and Anne puts the cookie in the basket. When Sally returns, the subjects are asked where Sally will look. According to people with a theory of mind, Sally will look in the box because she doesn't know that the cookie switched places. However, people without a theory of mind will say that Sally will look in the basket because they don't understand that Sally doesn't know exactly what they, the observer, do. To make decisions, theory-of-mind AI that can understand humans must be created. They will also be able to recognize emotions. AI has been shown to have the basic theory of mind, meaning that they can recognize the way other $\mathrm{AI}$ and human brains work.

The way many AI and machine learning algorithms work right now is based on a supervised model. For example, if an AI must recognize hand-written digits, it is given a series of pictures of handwritten digits, which are classified. However, things aren't always classified in the real world, and humans must make sense of raw data. This is currently one of the separations between AI and human brains. AI must be able to take raw input data and make sense out of it to achieve its goals. There is currently much development occurring in the unsupervised learning AI. This shows that it is most likely possible for AI to work without human support in the future.

If these two milestones, theory of mind and individual functionality, are achieved, then many more tasks could be done by AI. AI could make decisions on socializing, gaining new relationships and friendships. If AI managed to achieve a theory of mind, this is guaranteed to happen. In this scenario, it is possible that AI could determine how good of a fit someone would be in a dating app scenario and exactly how the relationship or friendship would go. Following the AI would almost guarantee a healthy and stable relationship with someone else since the AI can look at vast amounts of data that is put online. There will be even more data for the AI to learn from in the future, which will lead to smarter AIs. AI could also help people converse more productively. If the AI wants an individual to get to know somebody, then the AI can also help with the actual conversation and interaction. For example, when speaking, the AI could determine how someone feels about them and what types of words would work the best. With a theory of mind, the AI could easily use immense processing power to do this. There is much evidence that a theory of mind could be achieved in the future $[9,10]$.

AI could also help people in college applications [6]. College consulting is a multi-billion-dollar industry in the United States. As discussed previously, there are already certain programs that help people decide which majors or colleges to apply to. The future may bring additional advancements that could act as college consultants, analyzing huge amounts of publicly available data to determine which kind of students would get into which colleges. It can also determine what kind of extracurricular activities are suitable or what essays would help. Because AI has unsupervised learning abilities as well as a theory of mind, it could do this. However, it would be much more efficient if the future brings a completely AI-based college admission office as it has already started to do. AI is more predictable than human beings making the theory of mind ability of the AI more pronounced. Because such an AI would make the admissions process unfair, the admission office may try different methods to make sure its admission office is fair and more unpredictable. Eventually, an arms race between the AIs would develop, creating a Nash equilibrium where neither side can improve their chances. The ball has fallen into a stable state. This is a possibility of such a system.

AI could help determine what kinds of jobs or life paths an individual should take. If AI has higher computing power and access to data, it may find correlations in data that can determine which kind of people will prefer what paths in life and in careers. These types of AI can be similar to the college consulting AIs in which they determine ideal majors or 
jobs. These AIs could look at a person's personality and other details gathered from the internet to find out what types of jobs would be more suitable. If the computing power allows for rudimentary simulations, it can even tell an individual what exactly may happen in their lives if they choose a certain path. This type of AI would be similar to what human parents do with children in certain cultures.

In general, AI could make decisions for normal people in many ways if certain technological goals are achieved. These examples can be further subdivided into smaller examples. In general, those technological goals can be achieved based on current rates of development.

\subsection{What is the Opinion of Different People from Different Age Groups or Cultural Backgrounds on the Use of AI to Make Decisions on Both Small Daily Decisions and Larger Decisions on Career Paths and Relationships with Other People?}

Based on the survey, it was found that teenagers and adults are similar in their thoughts regarding free will and the use of AI to replace a free will. However, when zooming in on smaller decisions about restaurants, adults were more likely to use it. For the chess competition question, there were almost no affirmative answers except for a single teenager. This can be considered an outlier. In terms of career planning and college majors, teenagers were very opposed to using AI for this. Adults were more open to the idea. For the socialization and relationships question, they were both similarly opposed to the idea. Based on this, it can be concluded that Teenagers and Adults have similar levels of support for most forms of AI aside from the use in career paths and relationships.

Based on the survey, it was found that individuals with a Chinese background valued freedom and free will less than western individuals. However, they had similar levels of support for the use of AI in general, which were low. Those of Chinese background were more supportive of the use of AI in making small decisions. Both Chinese and Western people were in opposition to the use of AI for winning chess competitions. Chinese people were more welcome to the idea of using AI to help with career planning and life paths. They were all similar for the use of AI in helping with socializing and relationships.

It may be hypothesized that teenagers were opposed to the idea because adults may investigate the past as something which can't change and wish that they made better decisions. This may be why Ms. Tian said that people shouldn't take so many tangents and that they should spend their time on one thing and do it well. Teenagers see the future as something more fluid and expansive. They may believe that they want to try out many different things.

The reason why people are all opposed to the use of $\mathrm{AI}$ in decision making in social life is that people often find the fun in socializing.

The result of the cultural differences may be caused by the fact that Chinese people put less value on freedom in their culture while westerners value freedom. For example, the American government has put stricter restrictions on face recognition software and technology because it believes it infringes on privacy. While there is some opposition to its use in civilian circles, it is mostly accepted in China.

The amount of support for AI's use in social life was similar to the amount of support for AI in general, suggesting a connection between the two. It could be possible that people associate their overall decisions with social decisions. This requires further investigation.

\subsection{Given Current Trends, How May Humans React to AI Taking Away Free Will?}

These studies were referenced above in the "Paradox of Choice" section and were all done to help marketing initiatives. However, the conclusion can help determine the amount that humans may oppose the use of AI to make decisions for them. In the future, there will be more decisions humans need to make. This is also due to the access to more choices in their lives as social mobility increases. There may also be more additional choices from the cultural changes that are happening. As discussed in Barry Schwartz's "Paradox of Choice", decisions that used to be made by the family or the community or the workplace are now being made by the individual. The amount of choice overload is further compounded by the fact that humans may have less time to consider each decision and more information due to the internet and big data. As discussed in Chernev's meta-analysis, the ability of the decision maker to make the decisions and the complexity of choice are strong determiners of the amount of choice overload. In addition, because more things that an individual does will be published online, decisions will have more value and more importance as everyday decisions carry more weight.

Based on the trends shown by the survey, it may mean that there is a lot of disagreement on the use of this technology and that humans may need larger amounts of cultural change for it to be possible for AI to make decisions for humans.

Based on this, it may be determined that psychology shows us that the paradox of choice may have an effect and that humans may be able to adapt to having less choice in their lives. However, the jelly bean experiment 
shows that a small amount of choice will lead to much more satisfaction. This means that, in general, it may be preferred to have a smaller amount of choice and that AI may need to program in the illusion of choice.

\section{CONCLUSION}

The advent of AI will have large ramifications on people's lives, and as it becomes more advanced, it may begin guiding humans in their lives as advisors. However, humans may dislike this system as many humans believe that free will is important. This paper has discussed whether such ideas are possible and how humans may react to such a development. It has been concluded that AI will most likely be able to accomplish such a task as playing the game of life. It has been concluded that people may adapt to this new environment well, but a small amount of real or illusory choices will make people happier. This research may have ramifications on any $\mathrm{AI}$ which is used in an advisory way as the choice may need to be programmed into many programs to make customers happier. However, the lack of freedom shouldn't be a large problem. In the future, more research can be done into the ways AI may interact with each other or how humans may integrate their minds with AI. In addition, more research can be done into the ethical considerations of how humans value free will. While people may say that they value free will, breaking it down may show that people don't need free will to be happy if the result is satisfactory.

\section{ADDENDUM}

\section{Raw Data and Interview Notes}

\section{Interviews}

Four people were interviewed, and the summaries of the interviews are as follows.

Subject 1: Mr. Wagonfuhr

Age: 14

Cultural Background: Western, German-American

In general, this subject was a lot more thoughtful about the topic, asking a lot of clarifying questions. This subject appears to deny the fact that AI would be able to achieve this, often asking questions such as "How does it know that?". He is more utilitarian and wasn't opposed to the use of $\mathrm{AI}$ and is fine with others making decisions for him. However, after further digging, he was a lot more indecisive and, in the end, he said, "Ehh, who cares, I'll do what I feel like doing". This subject mentioned both the Thunderhead in the book Scythe and the book I Have No Mouth and I Must Scream. This subject isn't quite sure about whether he thinks AI taking free will help humanity or imprison humanity.

Subject 2: Mr. Sheng

Age: 15

Cultural Background: Chinese

In general, this subject wasn't opposed to the idea of AI taking away free will. However, for scenarios involving making friends and relationships, he was opposed. He was also opposed to the use of AI for chess because he thought that long term decisions should be done by AI, though he also thought that restaurants could be done by AI.

Subject 3: Ms. Tian

Age: 31

Cultural Background: Chinese

This subject had a lot of thoughts on the topic and spoke for over two hours. In general, this subject was more practical, mostly caring about AI taking away her "physical and financial security". In terms of playing chess, she was opposed to doing it and believed that AI can't do things for you, it can only cover you up. She often referenced "some people" in a negative light. She believes in practicality and that going on tangents because of personal interest is bad. She trusts technology more than other people. She is a STEM major as well as an investor in technology which may be the reason for this. She said, "I trust AI. I trust data. I trust logic."

\section{Subject 4: Mr. Luo}

Age: 25

Cultural Background: Western, American

This subject was more opposed to AI taking away free will. He said that people feel better when they have free will and that freedom is a fundamental way of living. However, when zooming in on the use of AI for restaurants, he often referred to using AI to narrow down choices instead of using it to have only one choice. In general, he believed that keeping choice or the illusion of choice was incredibly important. 


\section{Survey}

Table 1. The following information has the answers grouped by age and ethnicity. The numbers will represent the percentage of people who answered "Yes". There were eight adults and nine teenagers who took the survey. Of those eight adults and nine teenagers who took the survey, ten were Chinese and seven were Westerners.

\begin{tabular}{|c|c|c|c|c|c|}
\hline Questions & Chinese & Western & Teenager & Adults & Total \\
\hline Do you value free will and freedom? & 60 & 86 & 78 & 75 & 70 \\
\hline $\begin{array}{l}\text { If an AI could make decisions about your life for you and tell you what would happen } \\
\text { if you made a certain decision, would you use it? }\end{array}$ & 50 & 57 & 56 & 50 & 53 \\
\hline $\begin{array}{l}\text { Let's suppose that there was an AI that could tell you which restaurant you should go } \\
\text { to. Current apps only tell you if you want to eat Chinese food, the best Chinese food } \\
\text { places. However, this AI can tell you exactly what you want before you know } \\
\text { yourself. It would know based on all the data you give it from the internet and big } \\
\text { data. Would you use this? }\end{array}$ & 90 & 71 & 78 & 88 & 82 \\
\hline $\begin{array}{l}\text { Suppose you are at a chess competition. There is a technology which can tell you } \\
\text { through the glasses that you are wearing, which move would be the best based on a } \\
\text { chess engine. This would guarantee victory. Would you use such a device to win? }\end{array}$ & 10 & 0 & 11 & 0 & 5 \\
\hline $\begin{array}{l}\text { What if an AI could predict exactly which colleges or majors would give you the most } \\
\text { money or happiness in the future? This AI would have the ability to simulate what } \\
\text { would happen in the future. }\end{array}$ & 70 & 57 & 44 & 88 & 64 \\
\hline $\begin{array}{l}\text { What if an AI could tell you whether someone would be a good friend or partner? } \\
\text { This AI would be able to tell you what will probably happen in the future. }\end{array}$ & 50 & 57 & 56 & 50 & 53 \\
\hline
\end{tabular}

\section{Paradox of Choice Jelly Bean Case Study:}

\section{Raw Data and Processed Data:}

Table 2. The Effect of the Number of Choices Available to Individuals on their Ratings of their Satisfaction on a Scale of 1-10.

\begin{tabular}{ccccc}
\hline Trial \# & & & & \\
\hline \# of Choices & $\mathbf{1}$ & $\mathbf{2}$ & $\mathbf{3}$ & $\mathbf{4}$ \\
$\mathbf{1}$ & 5 & 3 & 4 & 5 \\
$\mathbf{2}$ & 5 & 7 & 7 & \\
$\mathbf{5}$ & 4 & 3 & 4 & \\
\hline
\end{tabular}

Table 3. Analysis of Table 2.

\begin{tabular}{lccc}
\hline \multicolumn{4}{c}{ \# of Choices Available } \\
\hline & Median & Mean & Range \\
$\mathbf{1}$ & 4.5 & 4.25 & 2 \\
$\mathbf{2}$ & 7 & 6.3 & 2 \\
$\mathbf{5}$ & 4 & 3.7 & 1 \\
\hline
\end{tabular}

2. Graph:

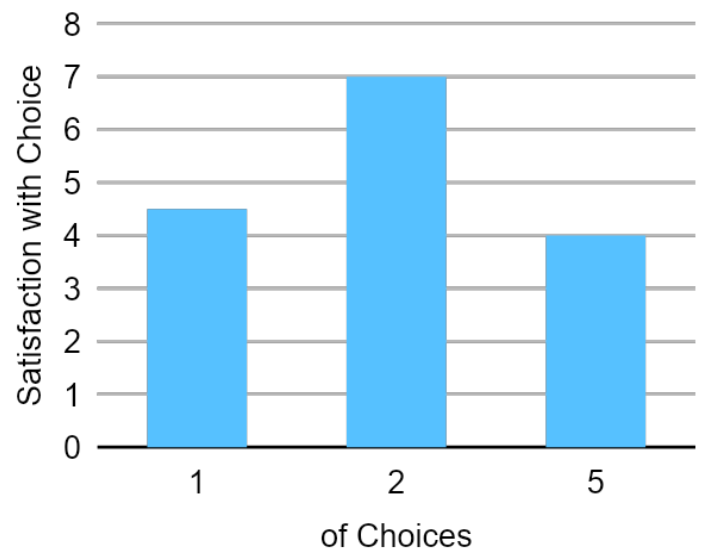

Figure 3 The Effect of the Number of Choices Available to Individuals on their Mean Ratings of their Satisfaction on a Scale of 1-10

\section{Analysis of Jelly Bean Experiment:}

Based on the above information, it can be concluded that a smaller amount of choice leads to a larger amount of satisfaction because having two choices far exceeds having one choice or having five choices.

\section{REFERENCES}

[1] "How to Cheat at Chess." Lichess.org, lichess.org/how-to-cheat. Accessed 9 June 2021.

[2] Schwartz, Barry (2004). "5". The Paradox of Choice. New York, United States: Harper Perennial. ISBN 0-06-000568-8.

[3] Iyengar, S. S., \& Lepper, M. R. (2000). When choice is demotivating: Can one desire too much of a good thing? Journal of Personality and Social Psychology, 79(6), 995-1006. https://doi.org/10.1037/0022-3514.79.6.995.

[4] Chernev, Alexander; Böckenholt, U.; Goodman, J.K. (2015). "Choice overload: A conceptual review and meta-analysis". Journal of Consumer Psychology. $25 \quad$ (2): $\quad 333-358$. doi:10.1016/j.jcps.2014.08.002.

[5] Hill, Kashmir. "How Target Figured out a Teen Girl Was Pregnant before Her Father Did." Forbes.com, 16 Feb. 2012, www.forbes.com/sites/kashmirhill/2012/02/16/how -target-figured-out-a-teen-girl-was-pregnantbefore-her-father-did/?sh=110e9e9b6668.

[6] DUMRONGKIAT MALA. "AI to Help Students Select Majors.” Bangkokpost.com, 27 Nov. 2018, 
www.bangkokpost.com/thailand/general/1582894/ ai-to-help-students-select-majors. Accessed 12 June 2021.

[7] Sakpal Manasi "Gartner Predicts $69 \%$ of Routine Work Currently Done by Managers will Be Fully Automated by 2024" 23 Jan 2020 https://www.gartner.com/en/newsroom/pressreleases/2020-01-23-gartner-predicts-69--ofroutine-work-currently-done-b.

[8] Baron-Cohen, Simon; Leslie, Alan M.; Frith, Uta (October 1985). "Does the autistic child have a "theory of mind"?". Cognition. 21 (1): 37- 46. doi:10.1016/0010-0277(85)90022-8. PMID 2934210. S2CID 14955234.Pdf.

[9] Wang, Zhiliang (2007). Smith, Michael J.; Salvendy, Gavriel (eds.). "Artificial Psychology". Human Interface and the Management of Information. Methods, Techniques and Tools in Information Design. Lecture Notes in Computer Science. Springer Berlin Heidelberg. 4557: 208217. doi:10.1007/978-3-540-73345-4_25. ISBN 9783540733454. S2CID 13060657.

[10] Winfield Alan F. T. (2018) Experiments in Artificial Theory of Mind: From Safety to StoryTelling Frontiers in Robotics and AI https://www.frontiersin.org/article/10.3389/frobt.2 018.00075 10.3389/frobt.2018.00075 ISBN 2296-9144. 